\title{
Excavation Sequence and Surrounding Rock Mass Stability of Large-Scale Underground Engineering with 8 Tunnels
}

\author{
Zhiqiang Zhang $\mathbb{D},{ }^{1}$ Mingming He $\mathbb{D},{ }^{1}$ Fangfang Chen, ${ }^{2}$ and Ning Li $\mathbb{D}^{1}$ \\ ${ }^{1}$ Institute of Geotechnical Engineering, Xi'an University of Technology, Xi'an 710048, China \\ ${ }^{2}$ School of Architecture and Civil Engineering, Xi'an University of Science and Technology, Xi'an, 710056, China \\ Correspondence should be addressed to Zhiqiang Zhang; zhangzq87@xaut.edu.cn
}

Received 21 February 2020; Revised 1 August 2020; Accepted 26 August 2020; Published 4 September 2020

Academic Editor: Hui Yao

Copyright (C) 2020 Zhiqiang Zhang et al. This is an open access article distributed under the Creative Commons Attribution License, which permits unrestricted use, distribution, and reproduction in any medium, provided the original work is properly cited.

\begin{abstract}
In some large-scale hydroelectric power projects, there are more than 3 tunnels that are too close to each other to eliminate the mutual influence during the excavation period, especially for large-scale tunnel groups. In this paper, aimed at analyzing the Bukun hydropower station consisting of 8 tunnels in Malaysia, the displacement, stress, and plastic zone of the surrounding rock mass are analyzed to study the effect of the excavation sequence on the stability of the surrounding rock mass for large-scale tunnel groups. On the one hand, the in situ monitoring of the surface displacement of the rock mass surrounding the tunnel using extensometers is performed to obtain the deformation characteristics on the excavation limit under the typical excavation sequence. On the other hand, a series of elastic-plastic 3D numerical experiments are carried out to explore the displacement characteristics, stability of the large-scale tunnel groups, and safety of the initial supporting system. The results show that the tendencies of the displacement increase corresponding to the tunnel face movement are similar for the three excavation sequences. The displacement under initial excavation sequence 2 (IES2) is the smallest among the three sequences; the area of the plastic zone under IES2 is the smallest among them; and the stresses in the shotcrete layer and axial forces in the rock bolt under the three excavation sequences are within the safety limitation. Initial excavation sequence 2 is an optimized excavation sequence, in which tunnels \#1 and \#5 are excavated first; after an advance of 3 times the diameter of the tunnel, tunnels \#3 and \#7 are excavated; tunnels \#2 and \#6 are excavated after an advance of 3 times diameters; and tunnels \#4 and \#8 are excavated after an advance of 3 times diameters.
\end{abstract}

\section{Introduction}

Tunnels are one of the main structures that allow water to flow from reservoirs to power plants in hydroelectric projects. There are always two or more power tunnels due to the electric generating unit in a large-scale project $[1,2]$. In some projects, there are so many power tunnels, their construction, such as excavation, often takes years to complete, and these tunnel excavations are the main time-consuming subproject in the entire project [3]. Many attempts have been made to minimize the influence of excavation on the stability of the surrounding rock mass in scheduled construction times $[4,5]$. In tunnel excavation applications, engineers and scholars are eager to find a good solution that can reduce the tunnel construction time to reduce project cost and improve the stability of tunnels, especially for large-scale tunnel groups. The excavation sequence is very important to the scheduled construction time and stability of large-scale tunnel groups. In this paper, a hydroelectric project with 8 power tunnels, the Bukun hydropower station in Malaysia, is selected to investigate the effect of excavation sequences on the stability of the rock mass surrounding the tunnel. The optimized excavation sequence is proposed based on the stability of the rock mass and the tunnel and the supporting system safety of largescale tunnel groups.

Regarding the mutual influence of tunnels during excavation, there are several reports on the influence of tunneling on the existing tunnels in the soil rock stratum using numerical or in situ monitoring methods, even by means of theoretical tools. A parametric study is performed based on 
numerical methods for a three-dimensional model to explore the complex interaction of the different parameters, which may affect the tunnel response on the open-pit mining sequence of existing tunnels [6]. In another example, the deformation of an existing tunnel is analyzed by 3D finite element methods (FEM), and the settlement of the overburden is obviously influenced by the structure of the existing tunnels through the plastic development of the soil layer $[7,8]$. An empirical method is developed to estimate the settlement trough caused by the second of twin tunnels, and it can provide a preliminary predictive tool for shield tunnels based on the Heathrow Express project in London, $\mathrm{UK}$, as well as a database of case histories for twin tunnels prepared from reported information worldwide [9]. In addition, a field monitoring application is presented to evaluate the close proximity tunneling influences of a new tunnel on an existing railroad tunnel. The principal component analysis-based monitoring framework is developed using the sensor datum collected from the existing tunnel while the new tunnel is under excavation [10]. A case of closely spaced twin tunnels excavated beneath other closely spaced existing twin tunnels is presented, and the settlements of the existing tunnels and the ground surfaces associated with the new tunnel construction were systematically monitored [11]. These results show that a close existing tunnel has a substantial influence on the newly constructed tunnel, and the new tunnel excavation also has a remarkable influence on the deformation and stress of the old tunnel in the surrounding rock mass. The failure of the support structure is the main problem for old tunnels. The stability of the surrounding rock mass is the main problem for newly constructed tunnels, especially for large-scale tunnel groups.

Physical modeling tests play an important role in studies related to the excavation of tunnels in soft ground. A variety of modeling techniques have been developed by researchers to study the ground response to tunneling worldwide [12]. Among the physical modeling test methods for geotechnical engineering, the centrifuge test is an important method in many studies. Some experts carried out research work by means of centrifuge model tests, and many results were achieved. A centrifuge model test was carried out to investigate the effect of new shield tunneling on the existing underlying large-diameter tunnel, and three construction steps of a new shield tunnel were simulated in this centrifuge model test [13]. The deformation and stress of the existing tunnel that was affected by the construction of new tunnels were systematically monitored. The settlement development of existing tunnels has four stages [14]. A series of threedimensional centrifuge model tests were conducted to investigate the effect of twin tunnel construction on the existing single pile in dry sand. The influence of the depth of each tunnel relative to the pile was investigated by constructing twin tunnels either close to the mid-depth of the pile shaft or near the pile toe [15]. Although centrifuge model testing is a suitable and available approach, there are very limited cases studying the effect of the large-scale tunnel group during the construction period.
In this paper, with the object of studying the influence of the excavation sequence on the stability of the rock mass surrounding a tunnel, a series of in situ monitoring tests of the rock mass surface displacement surrounding a tunnel using tape extensometers are carried out to obtain the deformation or displacement on the excavation limit under typical excavation sequences. In addition, to obtain more information on the surrounding rock mass and supporting system, a systemic series of elastic-plastic 3D numerical experiments are carried out by FEM to explore the deformation characteristics and stability of the rock mass surrounding the tunnel under different excavation sequences based on the Bukun hydropower station in eastern Malaysia. Finally, an optimized excavation sequence is suggested for the Bukun hydropower station.

\section{Overview of the Bukun Hydropower Station}

2.1. Location and Main Building. The Bukun hydropower station is located on the Balui River in eastern Malaysia, approximately $37 \mathrm{~km}$ from Belaga town and $180 \mathrm{~km}$ from port town Bintulu. This hydrojunction project consists of four main buildings: concrete-faced rockfill dams, tunnels and a powerhouse on the left bank, a discharge structure, and a diversion structure on the right bank. The elevation of the earth dam is $235.00 \mathrm{~m}$, the maximum height of the dam is 205.0 meters, and the total reservoir capacity is $44.0 \mathrm{Gm} 3$. The layout of the power tunnels is shown in Figure 1.

There are 8 tunnels that can introduce water from the reservoir to the powerhouse; the inner diameter of the tunnel is approximately 9.0 meters, and the tunnel cross-section is also present in Figure 1. The length of the tunnel is approximately 600 meters. The orientation of the tunnel axis is $\mathrm{NW} 341^{\circ}$, the overburden of the tunnel is in the range of $50-235 \mathrm{~m}$, and the diameter of the tunnel excavation limit is approximately 10 meters.

\subsection{General Geological Information of the Tunnel.} According to the geology report by Lahmeyer Limited Company, there are mainly three types of stratum in the area of concern, such as greywacke, shale, and interbedding of greywacke/shale. The main rock strata along the tunnel are shown in Figure 2. The surrounding rock mass at the power tunnel is predominantly slightly weathered $\sim$ fresh greywacke, shale (mudstone), and greywacke/shale interbeds, of which greywacke accounts for 50.0 62.6\% (with an average of $54.8 \%$ ), shale (mudstone) accounts for $30.9 \sim 43.0 \%$ (with an average of $38.4 \%$ ), and greywacke/shale interbeds account for approximately $4.5 \sim 9.2 \%$ (with an average of $6.8 \%$ ). The greywacke is thick-bedded with sparse joints, and bedding is poorly developed with a high strength, and it can be regarded as an isotropic rock mass. Shale (mudstone) and graywacke/shale interbeds are anisotropic due to obvious interlayer joints.

The surrounding rock mass is classified into 4 grades (from grades I to IV), and the geomechanical property parameters of each surrounding rock mass grade are shown in Table 1. 


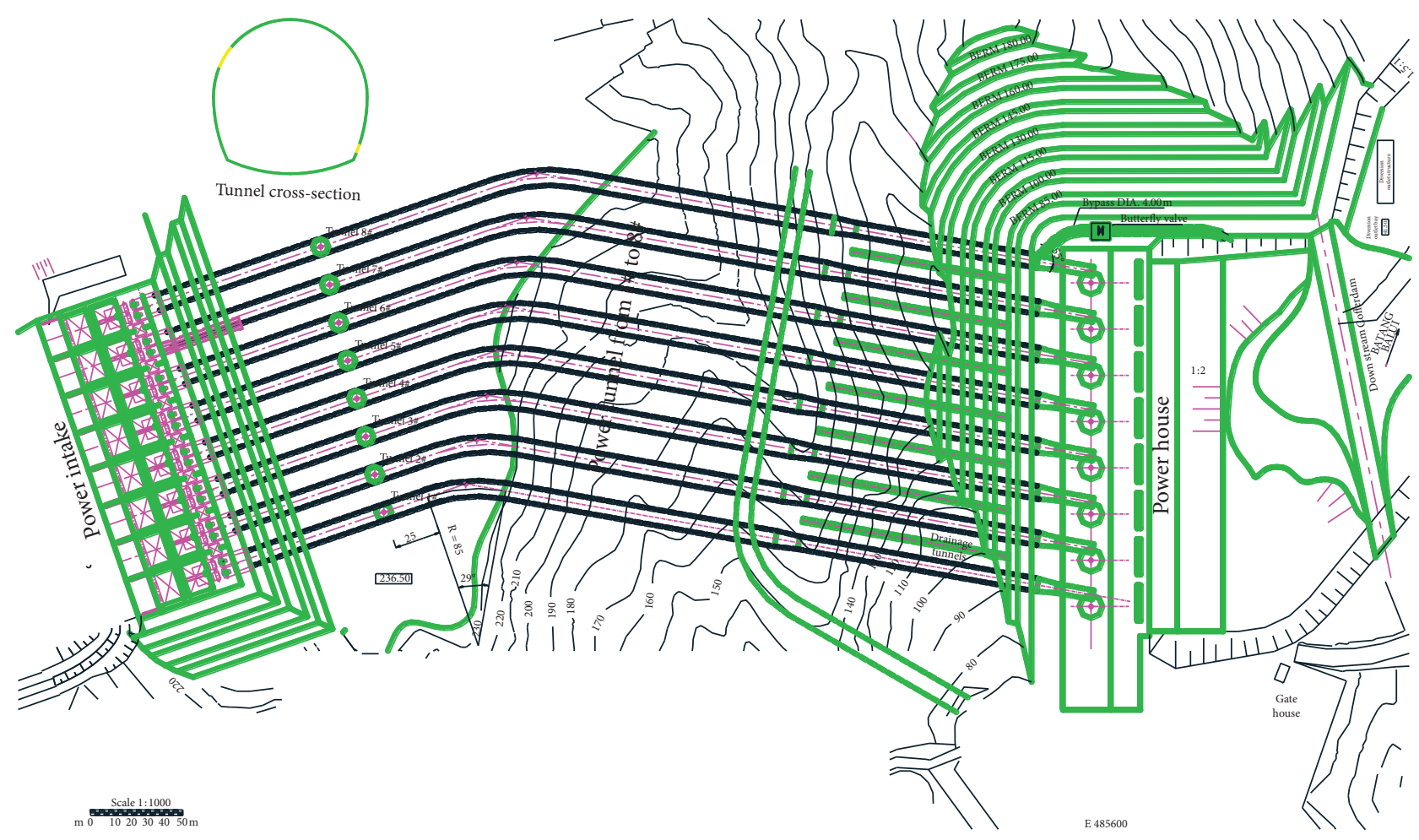

Figure 1: Layout of 8 power tunnels in the Bukun hydropower station and tunnel cross-section.

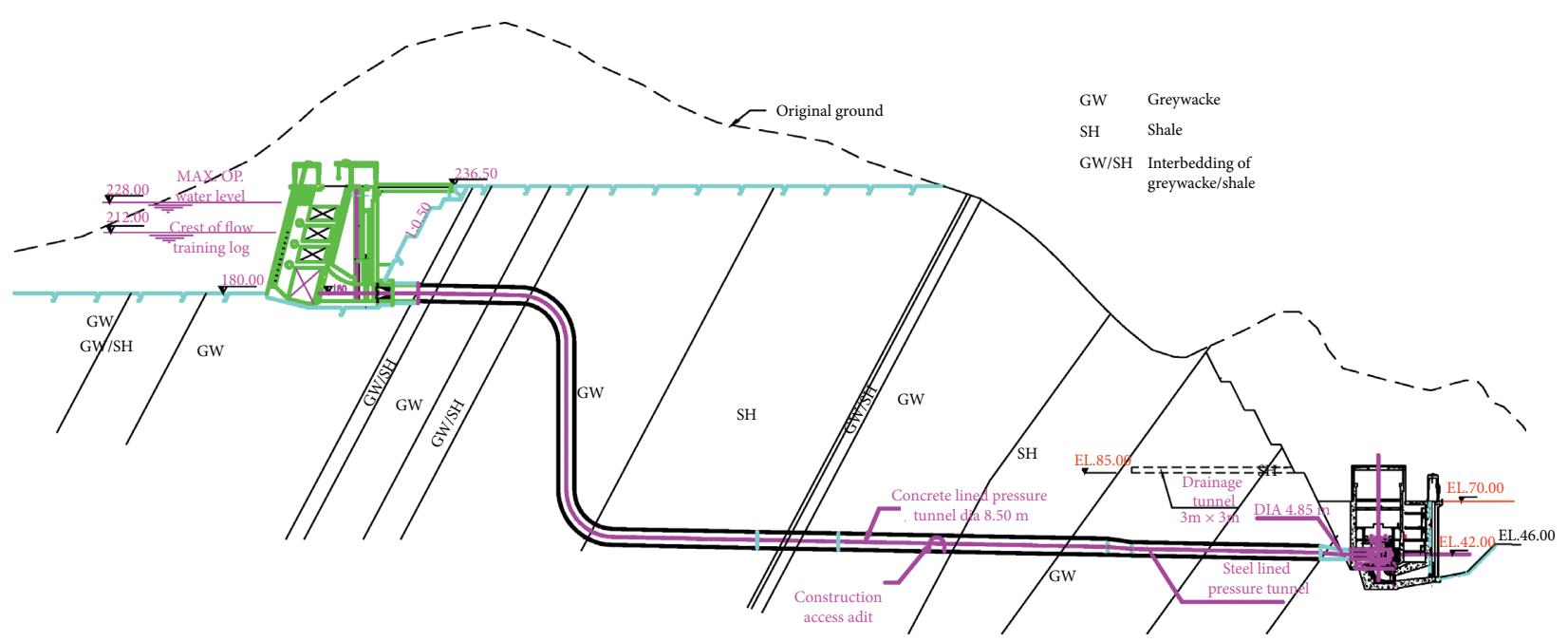

FIgURE 2: Longitudinal section of the power tunnel.

TABLE 1: Geomechanical property parameters of the surrounding rock mass.

\begin{tabular}{|c|c|c|c|c|c|c|}
\hline Grade & Density $\left(\mathrm{g} / \mathrm{cm}^{3}\right)$ & Poisson's ratio & Deformation modulus (GPa) & Friction coefficient & Cohesion (MPa) & Tensile strength $(\mathrm{MPa})$ \\
\hline I & 2.6 & 0.18 & $12.0 \sim 8.0$ & $1.20 \sim 1.10$ & $2.00 \sim 1.50$ & $1.80 \sim 1.20$ \\
\hline II & 2.6 & 0.20 & $8.0 \sim 5.0$ & $1.10 \sim 0.90$ & $1.50 \sim 1.00$ & $1.20 \sim 0.80$ \\
\hline III & 2.6 & 0.25 & $5.0 \sim 3.0$ & $0.80 \sim 0.75$ & $0.80 \sim 0.65$ & $0.64 \sim 0.52$ \\
\hline IV & 2.4 & 0.30 & $3.0 \sim 1.0$ & $0.70 \sim 0.65$ & $0.60 \sim 0.40$ & $0.48 \sim 0.32$ \\
\hline
\end{tabular}

2.3. Initial Excavation Sequence (IES). There are 8 power tunnels in the Bukun hydropower station, and a reasonable excavation sequence is important to shorten the construction time and improve the safety of the excavation. Three initial excavation sequences (IES1, IES2, and IES3) are initially suggested and are described further in Table 2.

Initial Excavation Sequences 1 (IES1): tunnels \#1 and \#3 are excavated first at the same time. Tunnels \#2 and \#4 are 
TABLE 2: Step of 3 IESs for 8 tunnels.

\begin{tabular}{lllr}
\hline Step & IES1 & IES2 & IES3 \\
\hline 1 & $\# 1$ and $\# 3$ & $\# 1$ and $\# 5$ & $\# 1, \# 3, \# 5$, and $\# 7$ \\
2 & $\# 2$ and $\# 4$ & $\# 3$ and $\# 7$ & $\# 2, \# 4, \# 6$, and $\# 8$ \\
3 & $\# 5$ and $\# 7$ & $\# 2$ and $\# 6$ & $\# 4$ and \#8 \\
4 & $\# 6$ and $\# 8$ & & \\
\hline
\end{tabular}

excavated after the faces of tunnels \#1 and \#3 move approximately 3 times the diameter; tunnels \#5 and \#7 are excavated after tunnel \#2, and the \#4 tunnel face moves approximately 3 times the diameter; tunnels \#6 and \#8 are excavated after tunnel \#5, and the face of tunnel \#7 moves approximately 3 times the diameter. IES1 is shown in Figure 3(a).

Initial Excavation Sequences 2 (IES2): tunnels \#1 and \#5 are excavated first at the same time, and then tunnels \#3 and $\# 7$ are excavated after tunnel \#1 and the \#5 tunnel face moves approximately 3 times the diameter; then, the tunnel faces of tunnels \#3 and \#7 move approximately 3 times their diameters, and tunnels \#2 and \#6 are excavated. The next step is for tunnels \#4 and \#8 to be excavated. IES2 is shown in Figure 3(b).

Initial Excavation Sequences 3 (IES3): tunnels \#1, \#3, \#5, and $\# 7$ are excavated first at the same time, and tunnels \#2, $\# 4$, \#6, and $\# 8$ are excavated after the $\# 3, \# 5$, and $\# 7$ tunnel faces move approximately 3 times their diameter. IES3 is shown in Figure 3(b).

The distance between the adjacent tunnels is 3 times the tunnel diameter. That is, the tunnel face of the next step is at a location of 3 times the tunnel diameter after the previous step, as shown in Figure 3.

\section{In Situ Monitoring}

3.1. Monitoring Scheme. On the construction site, basic displacement monitoring is conducted to obtain the deformation information of the rock mass surrounding the tunnel and evaluate the validation of the three IESs. Displacement monitoring is conducted using the tape extensometer tool. Three sections are selected to perform the monitoring in tunnels \#1\#4. Each section will be excavated by lengths of $50 \mathrm{~m}$ during monitoring, as shown in Figure 4.

In preparing the monitoring point, a measuring crosssection consists of three displacement rings, and a measuring set consists of five measuring cross-sections. Each measuring cross-section has an interval distance of 3 meters, as shown in Figure 5. All 3 displacement rings in the same crosssection are located in the same plane perpendicular to the tunnel axis. There are three measuring lines in a measuring cross-section. The length of the measuring line can be obtained by a tape extensometer. The vertical displacement of the top point and the convergence deformation of the two sidewalls can be calculated by three lengths of the measuring line based on the constant area of the triangle formed by three measuring lines. The supporting systems are the same in the three tunnel sections, and the shotcrete layer is type C20, with a thickness of $15 \mathrm{~cm}$, a rock bolt diameter of
$22 \mathrm{~mm}$, a length of $3.5 \mathrm{~m}$, and a row and column spacing of $1.0 \mathrm{~m} \times 1.5 \mathrm{~m}$.

The displacement measuring rings are installed $0.5 \mathrm{~m}$ behind the tunnel face. The first measurement is taken approximately 2 hours after installation. After the first measurement, the subsequent measurement is performed at each 3 meter distance ahead of the tunnel face.

3.2. Displacement Datum and Discussion. Based on the monitoring datum on tunnels \#1 to \#4, the effect of the IES on the deformation characteristics of the rock mass surrounding the tunnel is discussed. In this section, only the datum from tunnels \#1 and \#2 is chosen for discussion. These displacement curves at both the top point and sidewall in tunnels \#1 and \#2 under different IESs are shown in Figure 6. The full displacement curves from the surface monitoring can be roughly divided into two stages: the initial rapid growth stage when the tunnel face is close to the monitoring cross-section and the stable convergence stage when the monitoring cross-section is far from the tunnel face.

The displacements of both the top and sidewall points are dramatically affected by the IES in tunnel \#1, but the influence is not obvious in tunnel \#2. In tunnel \#1, the total displacements of the top points are $7.82,6.24$, and $8.51 \mathrm{~mm}$ from IES1 to IES3, respectively. The total displacements of the sidewall points are $6.33,5.15$, and $6.85 \mathrm{~mm}$. For tunnel $\# 2$, the top point displacements are $5.46,5.31$, and $5.48 \mathrm{~mm}$, respectively. The sidewall point displacements are 4.57, 4.45, and $4.59 \mathrm{~mm}$ from IES1 to IES3, respectively. From the displacement curves of the top and sidewall points in tunnel \#1, after installation of the displacement ring, the displacement increase is triggered by the advance of the tunnel and close tunnel face.

Comparing the displacements under the three IESs, the smallest displacement is produced under IES2, and the largest displacement is produced under IES3. Thus, IES2 is the best initial excavation sequence for the surrounding rock mass stability of the tunnel among the three IESs.

\section{Numerical Experiment}

4.1. Modeling. To obtain more information on the stability of the rock mass surrounding the tunnel during excavation, a series of 3D numerical experiments are carried out using the FEM code ABAQUS. The whole 3D model created by the ABAQUS preprocessor is shown in Figure 7(a). The element type used in the 3D model is C3D20. The model of 8 tunnels embedded in the whole $3 \mathrm{D}$ model is presented in Figure 7(b). 


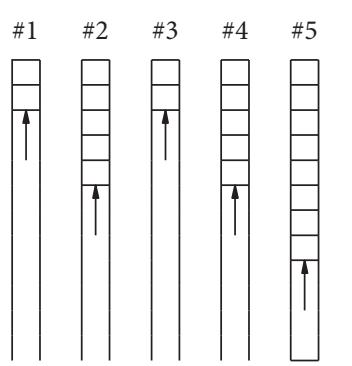

(a)

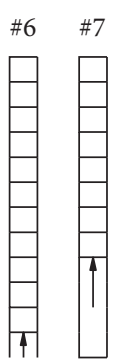

FIgURE 3: Schedule of 3 IESs for 8 tunnels: (a) IES1, (b) IES2, and (c) IES3.

(b)
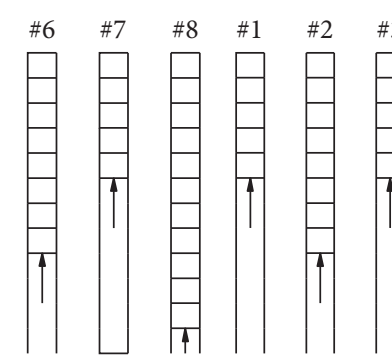

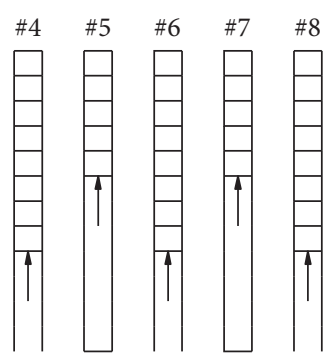

(c)

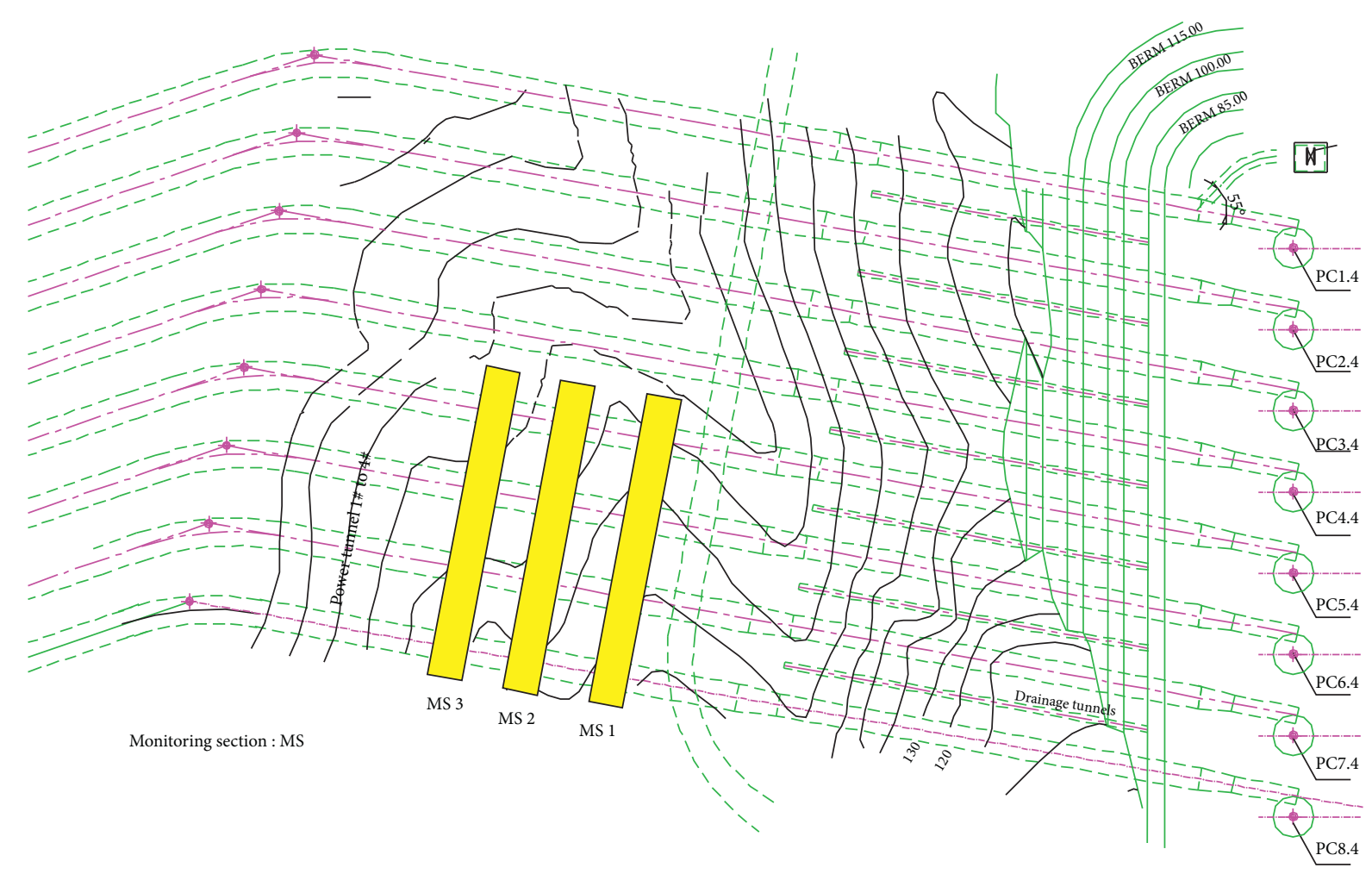

FIGURE 4: Three sections selected for in situ monitoring.

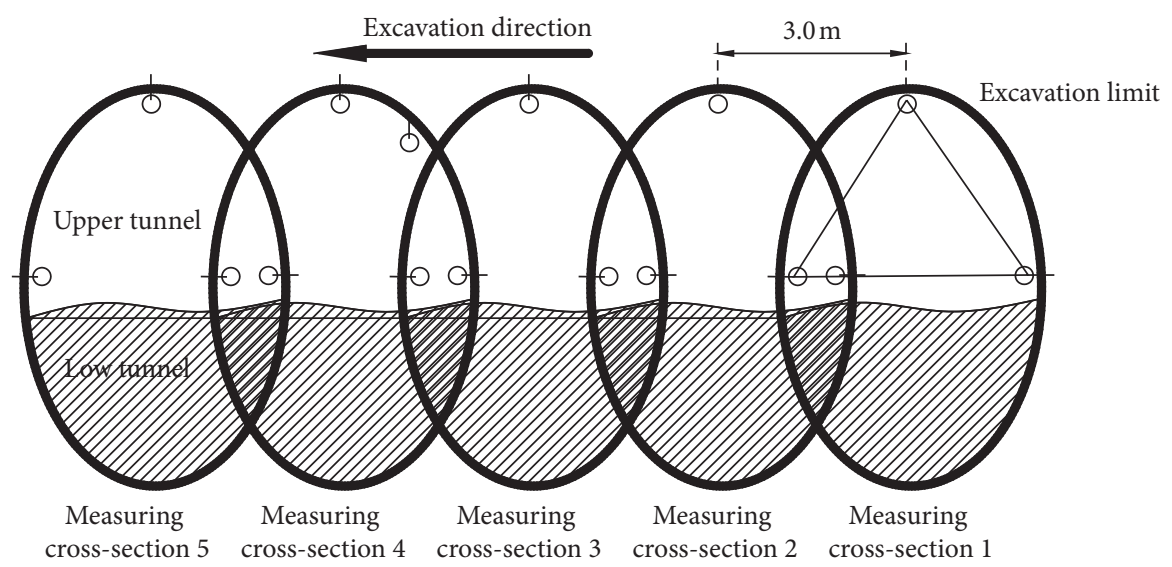

Figure 5: Measuring cross-section and displacement ring. 

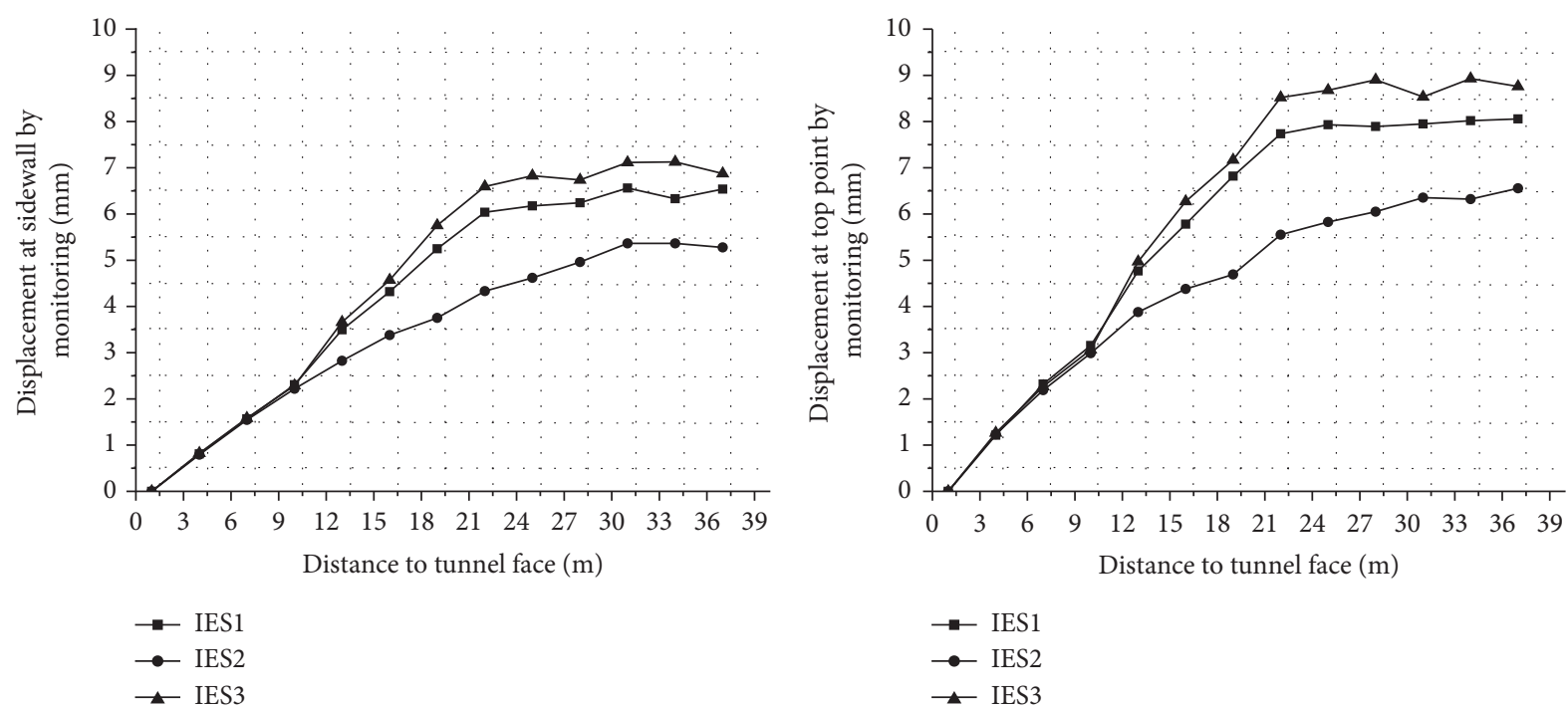

(a)
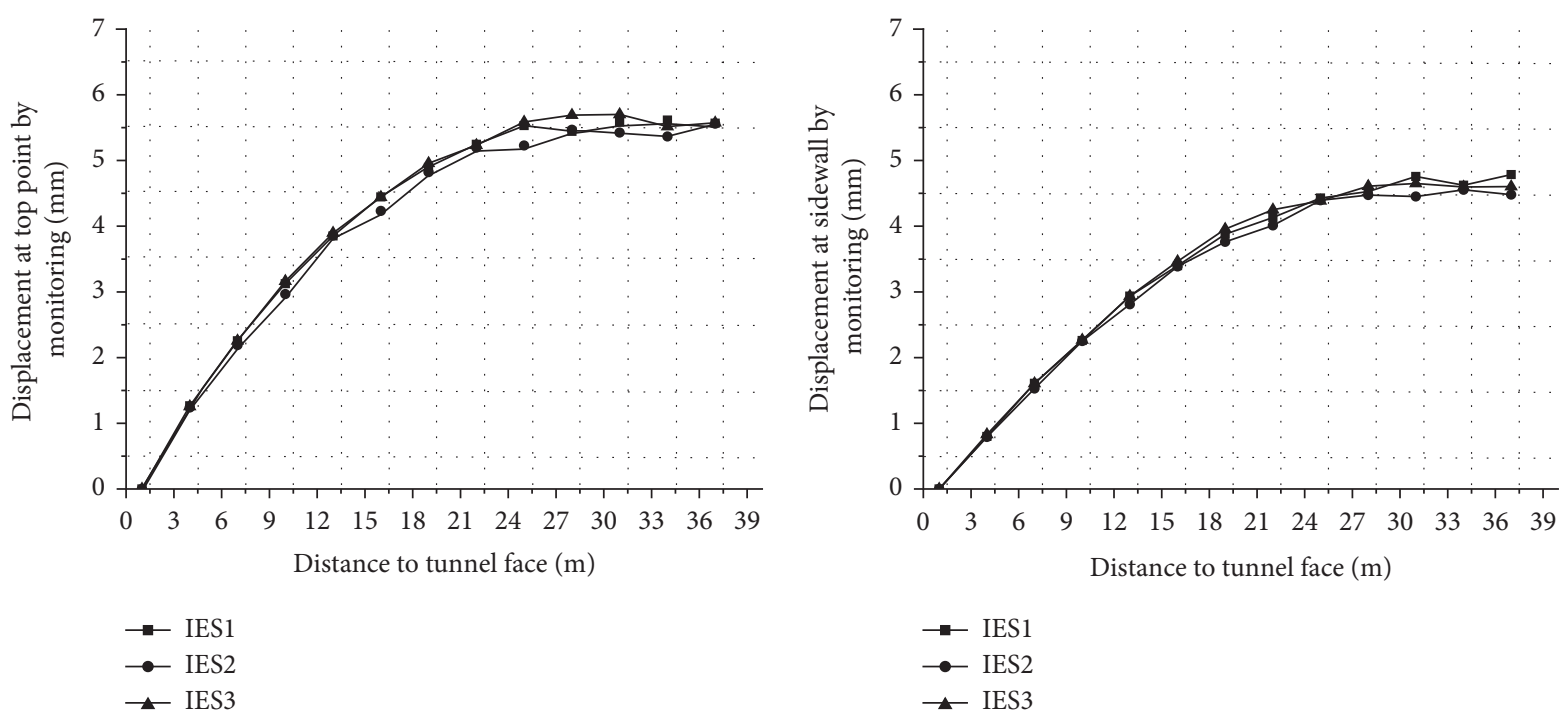

(c)

(d)

Figure 6: Measuring displacement curves of tunnels \# 1 and \#2 under different IESs: (a) top point on tunnel \#1, (b) sidewall point on tunnel $\# 1$, (c) top point on tunnel \#2, and (d) sidewall point on tunnel \#2.

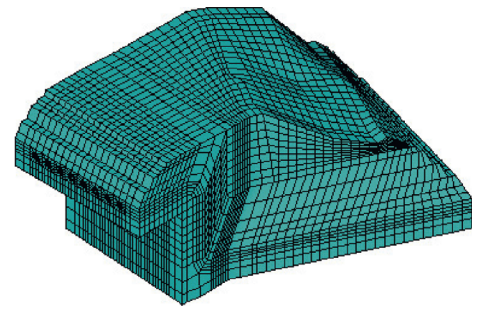

(a)

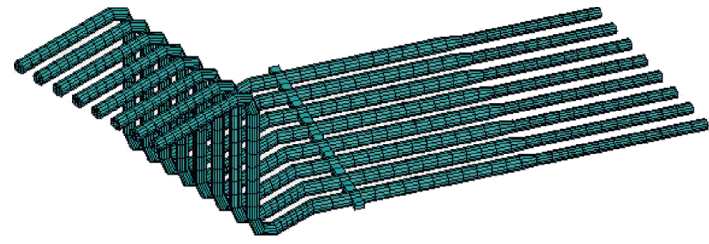

(b)

FIGURE 7: Geometry model for the 3D numerical experiment: (a) whole 3D model and (b) model 8 tunnels. 
The boundary of the whole 3D model includes the upper boundary, which is the original ground surface, and the bottom boundary, which is a plane with 3.0 times the tunnel diameter beneath the lowest tunnel. The two side boundaries are two planes that are located 2.5 times the tunnel diameter away from the tunnels.

In the numerical experiments, the surrounding rock mass of the tunnel is regarded as an elastic-plastic material with the Mohr-Column yield criterion. The material of the shotcrete layer and rock bolt is set as an elastic material. The physical and mechanical parameters of the rock mass, shotcrete, and bolt inputted into the numerical model are listed in Table 3.

According to a local geological report, the initial ground stress in the rock stratum around the project is a gravitydominated stress field. The vertical initial stress is expressed as $\sigma_{v}=\gamma H$. The horizontal initial stress is expressed as $\sigma_{h}=k_{0} \sigma_{v}$, where $H$ is the overburden of the rock stratum, $\gamma$ is the unit weight of the rock mass, and $k_{0}$ is the coefficient of the confining pressure in the rock stratum, where $k_{0}=(1-\mu) / \mu$.

To correctly simulate the excavation procession and response, the load releasing model is used to calculate the excavation loading on the tunnel limit, and it is applied in the numerical experiment as follows:

$$
\left\{\Delta F_{A}\right\}=\int_{S_{A}}[B](1-\alpha)\left\{\sigma_{0}\right\} \mathrm{d} A,
$$

where $\Delta F_{A}$ is the ground stress releasing load vector, $B$ is the element geometric matrix, $\alpha$ is the releasing coefficient of the stress, $\sigma_{0}$ is the initial ground stress vector, and $S_{A}$ is the area of the rock excavation.

The excavation releasing load is a function including some factors, such as the excavation sequence, supporting time, and advance of the tunnel face. The stress-releasing coefficient $\alpha$, a comprehensive index, can be obtained from engineering experience for a construction site. The initial excavation sequences applied in the numerical experiment are the same sequences as the in situ construction, IES1, IES2, and IES3.

4.2. Comparing the In Situ Monitoring Results. Due to the delay in the in situ monitoring from the tape extensometer in the tunnel, the displacement from in situ monitoring is only a portion of the whole displacement during the entire excavation period. In this section, the results of the numerical experiments and in situ monitoring under the same scenario are discussed. The point at the top of the tunnel is regarded as the representative point on the excavation limit, and the datum of three IESs is discussed. All the displacement curves from tunnel \#1 are plotted in Figure 8. In Figure 8, "-M" represents results obtained by monitoring, and "-N" represents results obtained by the numerical experiment.

By analyzing these displacement curves in Figure 8, it is evident that the tendency of the displacement increasing at the top point regarding to the tunnel face advance for all the curves is roughly similar by methods of both monitoring and numerical experiments. The displacement obtained by monitoring is smaller than that from the numerical experiment method. The similarities in the tendency and value of the displacement indicate that the numerical model in this paper is qualified to carry out further analysis.

\subsection{Complete Displacement Curves and Total Displacement.} The results can be obtained in the numerical experiments, such as the displacements and stresses at all the points, especially the complete displacement curves from the in situ monitoring. Three curves are presented in Figure 9, and all the displacements are the deformation datum at the top point under three IES cases for tunnel \#1.

Ahead of the tunnel face passing the monitoring crosssection, there is the same trend of the displacement increasing among the three IES cases. After the tunnel face passing through the monitoring cross-section, the displacements increase at different speeds at the top point under the three cases and reach different total displacements.

The displacements at the top point in tunnels \#1 \#8 are listed in Table 4. Under the same IES, the displacements at the top point in tunnels \#4 and \#5 are smaller than those in the other tunnels. By comparing the three IESs, the displacement under IES2 is the smallest. The displacement under case IES3 is the largest displacement among them. Therefore, according to the displacements of the surrounding rock mass at the top point, IES2 is a better excavation sequence for the stability of the rock mass surrounding the tunnel.

4.4. Plastic Zone of the Surrounding Rock Mass. The plastic zone is another index of the stability of the surrounding rock mass for tunnels. The plastic zones of the rock mass surrounding the tunnel under the three IESs are presented in Figure 10. The plastic zones are distributed mainly around the tunnel excavation limit in the surrounding rock mass near the tunnel bottom, sidewall, and crown. The main differences occur in the tunnel crown and sidewall area.

By comparing the area of the plastic zone, case IES2 has the smallest plastic zone among the three cases, and case IES3 is the largest one among the three cases. Therefore, case IES2 is a better choice for tunnel excavation.

4.5. Shotcrete Layer Stress. The maximum/minimum stresses at some specific location in the shotcrete layer are listed in Table 5. Under all IES cases, all the stresses in the shotcrete layer are compressive, which shows that all the shotcrete layers are under compressive stress conditions.

For one tunnel, the maximum compressive stress appears at the middle point in both sidewalls. For all the tunnels, these stresses in number \#4 or \#5 are larger than those of the other tunnels at the same location in the shotcrete layer for the same IES. In detail, for case IES1, the maximum stresses are 7.33, $7.44,12.53,10.09,7.30,11.48$, and $11.21 \mathrm{MPa}$ for tunnels \#1 to $\# 8$ in the shotcrete layer, respectively. The maximum stresses are 9.54, 9.61, 9.12, 7.12, 9.02, 10, 9.09, and $9.01 \mathrm{MPa}$ for 8 tunnels under case IES2, respectively. The maximum stresses are $7.22,7.35,12.17,7.61,12.28,7.52,11.62$, and $11.49 \mathrm{MPa}$ for 8 tunnels under case IES3, respectively. 
TABle 3: Physical and mechanical parameters of the rock mass, shotcrete, and bolt.

\begin{tabular}{lcccccc}
\hline Material & Density $\left(\mathrm{g} / \mathrm{cm}^{3}\right)$ & Poisson's ratio & Deformation modulus $(\mathrm{GPa})$ & Friction coefficient & Cohesion $(\mathrm{MPa})$ & Tensile strength $(\mathrm{MPa})$ \\
\hline Rock II & 2.6 & 0.20 & 6.0 & 1.0 & 1.2 & 1.0 \\
Rock III & 2.6 & 0.25 & 4.0 & 0.8 & 0.8 & 0.6 \\
Rock IV & 2.4 & 0.30 & 2.0 & 0.7 & 0.5 & 0.45 \\
Shotcrete & 2.4 & 0.167 & 210.0 & & 1.1 \\
Bolt steel & 7.8 & 0.30 & & & 310.0 \\
\hline
\end{tabular}

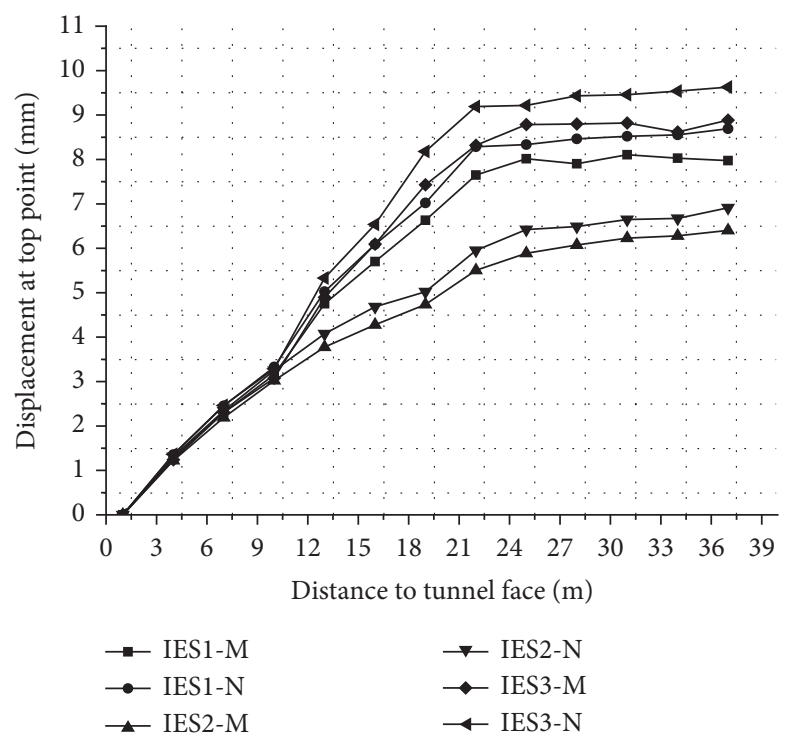

FIGURE 8: Displacement curves of the top point obtained by monitoring and numerical simulations.

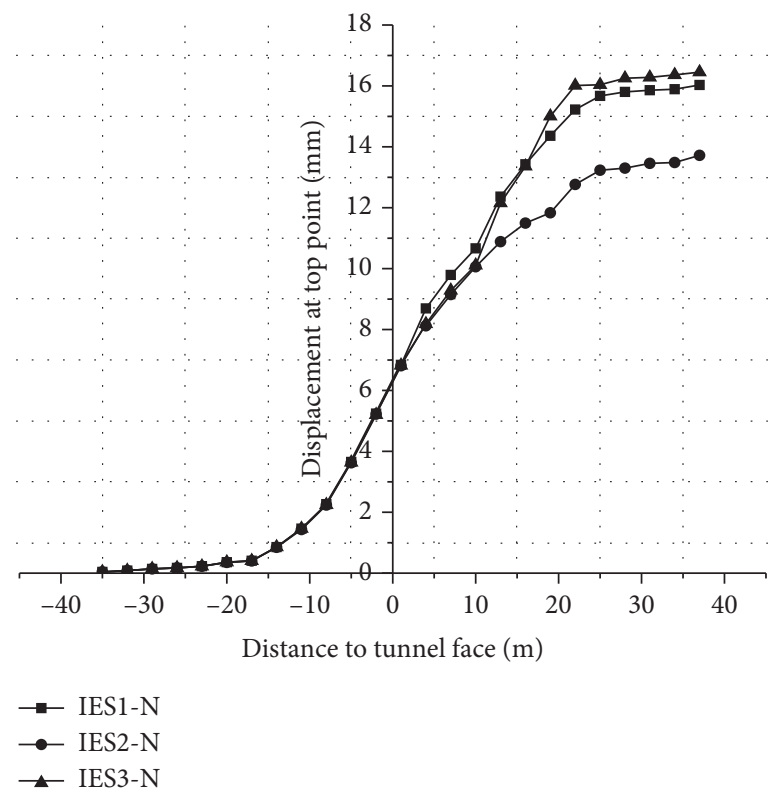

Figure 9: Three curves of the displacement at the top point of tunnel $\# 1$.

In the three IES cases, the maximum stress in the shotcrete layer is $12.53 \mathrm{MPa}$, and it is induced at the left sidewall point in tunnel \#3 under case IES1. All the stresses in the shotcrete layer are less than the compressive strength of the shotcrete. Any one of three IES cases can be a choice for the excavation sequence designing only considering the stresses in shotcrete layer. 
TABLE 4: Total displacements at the top point under different IESs.

\begin{tabular}{lcccccc}
\hline \multirow{2}{*}{ Tunnel } & \multicolumn{2}{c}{ IES1 } & \multicolumn{2}{c}{ IES2 } & \multicolumn{2}{c}{ IES3 } \\
& Horizontal/(mm) & Vertical/(mm) & Horizontal/(mm) & Vertical/(mm) & Horizontal/(mm) & Vertical/(mm) \\
\hline$\# 1$ & 0.46 & 16.43 & 0.33 & 13.72 & 0.43 & 16.71 \\
$\# 2$ & 0.45 & 16.76 & 0.39 & 14.14 & 0.46 & 17.58 \\
$\# 3$ & 0.21 & 17.50 & 0.21 & 14.97 & 0.25 & 18.37 \\
$\# 4$ & 0.00 & 17.98 & 0.06 & 15.36 & -0.07 & 18.96 \\
$\# 5$ & -0.15 & 18.01 & -0.09 & 14.91 & -0.25 & 18.77 \\
$\# 6$ & -0.28 & 17.77 & -0.28 & 14.15 & -0.46 & 17.58 \\
$\# 7$ & -0.48 & 16.61 & -0.51 & 13.44 & -0.48 & 17.03 \\
\hline 8 & -0.48 & 16.40 & -0.49 & & & \\
\hline
\end{tabular}

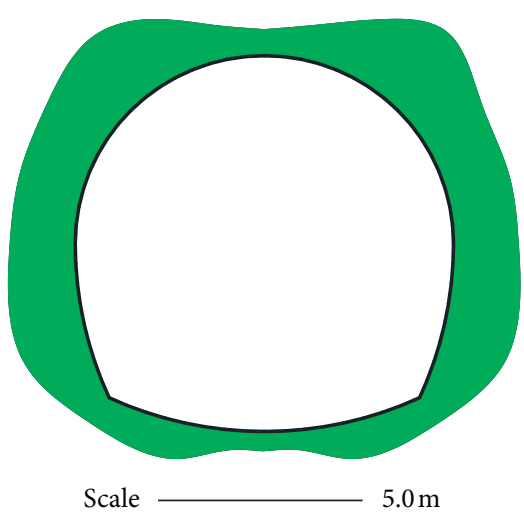

(a)

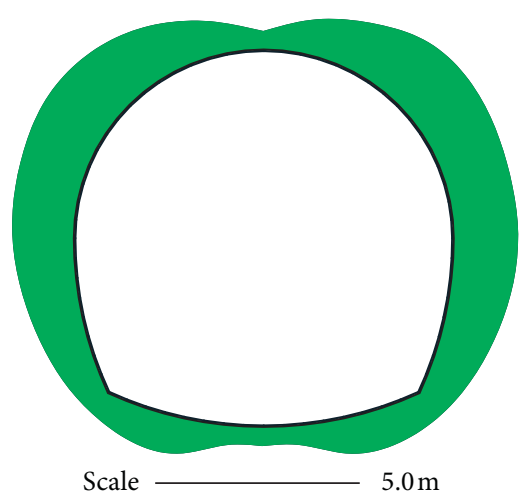

(b)

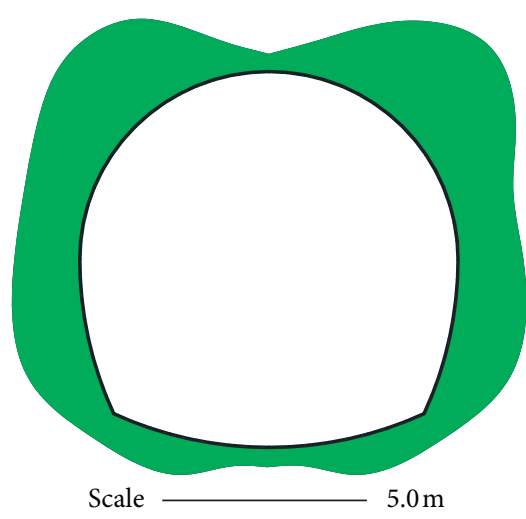

(c)

FIgURe 10: Plastic zone under three IESs: (a) IES1, (b) IES2, and (c) IES3.

TABLE 5: Stress at the top and left sidewall points in the shotcrete layer.

\begin{tabular}{|c|c|c|c|c|c|c|c|}
\hline \multirow{2}{*}{ Tunnel } & \multirow{2}{*}{ Location } & \multicolumn{2}{|c|}{ IES1 } & \multicolumn{2}{|c|}{ IES2 } & \multicolumn{2}{|c|}{ IES3 } \\
\hline & & Min. (MPa) & Max. (MPa) & Min. (MPa) & Max. (MPa) & Min. (MPa) & Max. (MPa) \\
\hline \multirow{2}{*}{$\# 1$} & Left & 6.79 & 7.33 & 8.74 & 9.54 & 6.76 & 7.22 \\
\hline & Top & 1.01 & 1.64 & 1.19 & 1.99 & 0.89 & 1.51 \\
\hline \multirow{2}{*}{$\# 2$} & Left & 6.85 & 7.44 & 8.87 & 9.61 & 6.78 & 7.35 \\
\hline & Top & 1.03 & 1.67 & 1.21 & 2.01 & 0.91 & 1.53 \\
\hline \multirow{2}{*}{$\# 3$} & Left & 11.77 & 12.53 & 8.43 & 9.12 & 11.44 & 12.17 \\
\hline & Top & 1.29 & 2.12 & 0.94 & 1.70 & 1.19 & 1.99 \\
\hline \multirow{2}{*}{$\# 4$} & Left & 9.51 & 10.09 & 6.74 & 7.12 & 7.21 & 7.61 \\
\hline & Top & 1.28 & 2.08 & 0.77 & 1.35 & 0.91 & 1.50 \\
\hline \multirow{2}{*}{$\# 5$} & Left & 8.87 & 9.42 & 8.48 & 9.02 & 11.77 & 12.28 \\
\hline & Top & 1.07 & 1.86 & 1.36 & 2.15 & 1.25 & 2.02 \\
\hline \multirow{2}{*}{ \#6 } & Left & 6.87 & 7.30 & 9.83 & 10.00 & 7.09 & 7.52 \\
\hline & Top & 0.87 & 1.33 & 1.37 & 1.97 & 0.93 & 1.39 \\
\hline \multirow{2}{*}{ \#7 } & Left & 10.88 & 11.48 & 8.49 & 9.09 & 11.03 & 11.62 \\
\hline & Top & 0.99 & 1.69 & 1.09 & 1.76 & 1.13 & 1.80 \\
\hline \multirow{2}{*}{$\# 8$} & Left & 10.57 & 11.21 & 8.31 & 9.01 & 10.93 & 11.49 \\
\hline & Top & 0.96 & 1.67 & 1.08 & 1.75 & 1.10 & 1.77 \\
\hline
\end{tabular}

4.6. Axial Force in the Bolt. To maintain a stable situation for the rock mass surrounding tunnels, a rock bolt is used as an important supporting structure working with the shotcrete layer. The axial forces at the sidewall range from 30 to $40 \mathrm{kN}$, and the axial forces at the top point range from 50 to $65 \mathrm{kN}$. All the axial forces of the rock bolt at the top point from tunnel \#1 to \#8 are listed in Table 6.
Under cases IES1 and IES3, both the maximum axial forces at the top point are approximately $65 \mathrm{kN}$, which occurs in tunnel \#6, but under case IES2, the maximum axial force is $62.5 \mathrm{kN}$. There is no apparent difference in the amount of bolt axial force among the three IES cases. All the maximum axial forces in any part of the surrounding rock mass are under the bearing capacity of the rock bolt. Hence, 
TABLe 6: Tensile axial forces in the bolts at the top point.

\begin{tabular}{lccc}
\hline Tunnel & IES1/(kN) & IES2/(kN) & IES3 $/(\mathrm{kN})$ \\
\hline$\# 1$ & 54.1 & 50.3 & 53.9 \\
$\# 2$ & 58.9 & 54.2 & 59.0 \\
$\# 3$ & 51.7 & 56.0 & 52.7 \\
$\# 4$ & 58.0 & 62.5 & 62.9 \\
$\# 5$ & 58.9 & 53.5 & 55.5 \\
$\# 6$ & 64.7 & 59.1 & 64.9 \\
$\# 7$ & 54.4 & 57.3 & 54.2 \\
$\# 8$ & 51.5 & 54.1 & 50.4 \\
\hline
\end{tabular}

all IESs can be a good choice for the excavation sequence design in consideration of the axial forces in the rock bolt.

For large-scale engineering with 3 or more tunnels, which tunnel is excavated first and which tunnel is excavated last are an important consideration. The excavation sequence for tunnels is an important factor in approaching a safe and economical plan. For a good excavation sequence, the most important requirement is to ensure the stability of the rock mass surrounding the tunnel and the safety of the supporting system. By comparing these results of systematic numerical experiments among cases IES1 to IES3, the safety of the supporting system can be assured by all IESs. An IES can be achieved. In our work, by considering the displacement and plastic zone, case IES2 is better optimized than IES1 or IES3. Therefore, IES2 is a better excavation sequence for the Bukun project with 8 tunnels.

\section{Conclusions}

Based on in situ monitoring and systemic numerical experiments, the stability of the rock mass surrounding the tunnel and safety of the supporting system are studied in this paper. The following conclusions can be drawn:

(1) By comparing the results from both monitoring and numerical experiments, although the displacement obtained by monitoring is smaller than that via numerical experiments, the similarity in the increasing tendency shows that the numerical model is qualified to carry out further analysis.

(2) In the displacement and plastic zone, the tendencies of the displacement increase corresponding to tunnel face movement are similar for the three IESs, but the total amount of displacement is different. The displacement under IES2 is the smallest among them. In the plastic zone, IES2 is the smallest case, and IES3 is the largest case among the three cases. ES2 is the better excavation sequence for the surrounding rock mass stability of the tunnel.

(3) The maximum stresses in the shotcrete layer are $12.53,9.61$, and $12.28 \mathrm{MPa}$ for the three IESs, respectively. All the stresses in the shotcrete layer are less than the compressive strength of the shotcrete. All IESs can be a choice for the excavation sequence in consideration of the stress in the shotcrete layer.

(4) The initial excavation sequence 2 (IES2) is recommended for engineering designers and owners. In
IES2, \#1 and \#5 are excavated first; after an advance of 3 times the diameter of the tunnel, tunnels \#3 and $\# 7$ are excavated; tunnels \#2 and \#6 are excavated after an advance of 3 times diameters; and tunnels \#4 and $\# 8$ are excavated after an advance of 3 times diameters.

\section{Data Availability}

The data used to support the findings of this study are included within the article.

\section{Conflicts of Interest}

The authors declare no conflicts of interest.

\section{Acknowledgments}

The scientific research program was supported by the $\mathrm{Na}$ tional Natural Science Foundation of China (11872301), Natural Science Basic Research Program of Shaanxi (2020JM-453), and Shaanxi Provincial Education Department Researching Foundation (17JS091).

\section{References}

[1] G.-L. Feng, X.-T. Feng, B.-R. Chen, Y.-X. Xiao, and Z.-N. Zhao, "Effects of structural planes on the microseismicity associated with rockburst development processes in deep tunnels of the jinping-II hydropower station, China," Tunnelling and Underground Space Technology, vol. 84, no. 2, pp. 273-280, 2019.

[2] G. Feng, X. Feng, B. Chen et al., "Characteristics of microseismicity during breakthrough in deep tunnels: case study of jinping-II hydropower station in China," International Journal of Geomechanics, vol. 20, no. 2, pp. 0401-0414, 2020.

[3] M. Nabipour, S. Rezapour, and S. H. Mohajeri, "A parametric study on friction-loss in water conveyance tunnels considering misalignment of precast concrete segments," Tunnelling and Underground Space Technology, vol. 96, no. 1, pp. 103$1015,2020$.

[4] W. Schubert and A. Steindorfer, "Selective displacement monitoring during tunnel excavation," Felsbau, vol. 14, no. 2, pp. 93-97, 1996.

[5] K. Zhao, M. Janutolo, and G. Barla, "A completely 3D model for the simulation of mechanized tunnel excavation," Rock Mechanics and Rock Engineering, vol. 45, no. 4, pp. 475-497, 2012.

[6] A. R. Beyabanaki and V. Gall, "3D numerical parametric study of the influence of open-pit mining sequence on existing tunnels," International Journal Of Mining Science and Technology, vol. 27, no. 3, pp. 459-466, 2017.

[7] M. Yin, H. Jiang, Y. Jiang, Z. Sun, and Q. Wu, "Effect of the excavation clearance of an under-crossing shield tunnel on existing shield tunnels," Tunnelling and Underground Space Technology, vol. 78, pp. 245-258, 2018.

[8] Q. Jiang, G. Su, X. T. Feng et al., "Excavation optimization and stability analysis for large underground caverns under high geostress: a case study of the Chinese laxiwa project," Rock Mechanics and Rock Engineering, vol. 52, no. 4, pp. 895-915, 2018.

[9] M. L. Cooper, D. N. Chapman, C. D. F. Rogers, and W. Hansmire, "Prediction of settlement in existing tunnel 
caused by the second of twin tunnels," Transportation Research Record: Journal of the Transportation Research Board, vol. 1814, no. 1, pp. 103-111, 2002.

[10] H.-B. Yun, S.-H. Park, N. Mehdawi et al., "Monitoring for close proximity tunneling effects on an existing tunnel using principal component analysis technique with limited sensor data," Tunnelling and Underground Space Technology, vol. 43, pp. 398-412, 2014.

[11] Q. Fang, D. Zhang, Q. Li, and L. N. Y. Wong, "Effects of twin tunnels construction beneath existing shield-driven twin tunnels," Tunnelling and Underground Space Technology, vol. 45, pp. 128-137, 2015.

[12] M. A. Meguid, O. Saada, M. A. Nunes, and J. Mattar, "Physical modeling of tunnels in soft ground: a review," Tunnelling and Underground Space Technology, vol. 23, no. 2, pp. 185-198, 2008.

[13] P. Li, S.-J. Du, X.-F. Ma, Z.-Y. Yin, and S.-L. Shen, "Centrifuge investigation into the effect of new shield tunnelling on an existing underlying large-diameter tunnel," Tunnelling and Underground Space Technology, vol. 42, pp. 59-66, 2014.

[14] R.-P. Chen, X.-T. Lin, X. Kang et al., "Deformation and stress characteristics of existing twin tunnels induced by closedistance EPBS under-crossing," Tunnelling and Underground Space Technology, vol. 82, pp. 468-481, 2018.

[15] C. W. W. Ng, H. Lu, and S. Y. Peng, "Three-dimensional centrifuge modelling of the effects of twin tunnelling on an existing pile," Tunnelling and Underground Space Technology, vol. 35, pp. 189-199, 2013. 